\title{
Improved executive functioning in healthy older adults after multifactorial cognitive training targeting controlled
} processes

\author{
Chambon $C^{*}$ and Alescio-Lautier B \\ Aix Marseille Univ, CNRS, LNSC, FR3C, Marseille, France
}

\begin{abstract}
Among the different cognitive training approaches tested in healthy older adults, multifactorial programs, which target higher-order processes such as memory and attention, best promote the transfer of learning to untrained cognitive processes.

Objective: In the present study, we investigated the effects of a multifactorial cognitive training program on executive functions in older adults.

Method: This training was developed to embed the processing of executive information in memory and attention training tasks, and mnemonic and executive strategies were implemented progressively throughout training.

Results: The results showed that this training program ( 24 sessions, 2 sessions per week) improved executive functions in older adults ( $\mathrm{n}=15$; mean age $=74.5 \pm 2.1$ ). Significant improvements were found for the switching and inhibition of irrelevant stimuli. We also report enhanced performance in the efficiency of a dual-task activity. Additionally, we report that these benefits were present at the 6-month follow-up without any additional training.
\end{abstract}

Conclusion: Taken together with previous results obtained with the same training protocol, the present results showed that the multifactorial training program we developed is an efficient tool to positively act on memory-related and executive aspects of the cognitive decline found in normal aging.

\section{Introduction}

Among the different kind of cognitive training programs existing to combat age-related declines in mental functioning, multifactorial ones have been developed to train memory and other functions, such as attention. Some data have shown that memory performances could be further improved with no transfer to other cognitive tasks [1-4], whereas other results have shown a transfer to other cognitive abilities such as language [5] and have enhanced nonspecific broad cognitive abilities [6]. In a previous study, we reported benefits in older adults after a multifactorial memory and attention training program in which 1) we embedded executive mechanisms in all training tasks, 2) mnemonic and executive strategies were implemented progressively throughout the training based on difficulties encountered by the subject, and 3) controlled processing at encoding was emphasized to allow the subject to cope with the increasing mental and attentional load throughout training while maintaining a good performance. Such training allowed improvement of recognition performances and near transfer of benefits to recall performance. The largest benefits were observed for tasks with high mental load. In addition, far transfer was observed with better memory self-perception and self-esteem of the participants [7]. Literature data thus clearly state that the multifactorial approach best promotes transfer to untrained cognitive processes.

In addition to studies investigating memory and attention deficit in normal aging, many studies focused on executive functioning in older adults. Executive function is used when we make a decision, plan an action, or adapt to a new situation $[8,9]$. It is thus seen as a set of controlled cognitive processes that play the role of the central administrator of many different cognitive mechanisms, including memory and attentional capacities, and which is crucial for goaldirected cognitive activities. Executive functions are heterogeneously affected during aging. Planning is affected, which could be related to shifting and updating deficits [10]. Whereas others have reported that younger adults recruited both working memory and fluid reasoning when performing a planning task, the older adult subjects mostly relied on their working memory capacity [11]. Attentional sharing, is also affected, especially when the older adults subjects were asked to perform a dual-task paradigm (for review see [12]). Switching has also been reported to be age sensitive $[13,14]$. The resistance to interference, which is linked to the ability to inhibit nonrelevant stimuli in a given context, was also shown to be age sensitive when tested in the Stroop interference test [15-18]. According to some of these authors, this agerelated effect must involve a breakdown in inhibitory processing. Such an explanation seems to be coherent with data reporting an effect of aging on inhibition as evaluated in many different tasks $[19,20]$.

${ }^{*}$ Correspondence to: Caroline Chambon, Laboratoire de Neurosciences Sensorielles et Cognitives - UMR 7260, Aix Marseille Univ \& Centre National de la Recherche Scientifique (CNRS), Centre Saint-Charles, Fédération de Recherche 3C - Case B, 3, Place Victor Hugo, 13331 Marseille Cedex 03, France, E-mail: caroline.chambon@univ-amu.fr

Key words: older adults, multifactorial cognitive training program, switching, resistance to interference, dual-task, executive function, rehabilitation

Received: November 12, 2019; Accepted: November 28, 2019; Published: December 02, 2019 
Given that 1) memory and attention abilities are tightly dependent on efficient executive functioning, 2) executive function is impaired in older adult individuals, and 3) memory benefits in older adult individuals are observed after our multifactorial training program in which we embedded executive mechanisms, we think that the memory improvement previously reported may be related to executive improvements. Therefore, we hypothesized that our multifactorial training program may have benefits on executive functioning in an older adult population. In the present study, we thus aimed to identify the benefits of our training program on many executive functions.

We investigated the capacity to resist to interference because we introduced distractors during our memory-training tasks and thus expected an improvement in this capacity. Mental flexibility is crucial during mental processes such as recognition, which implies comparing a current element to elements that are stored in memory to answer whether this current element is one previously observed, or during the recall process, which implies searching in memory for a precise element to provide a correct answer. Therefore, training recognition as we did in our training program may have had a beneficial effect on flexibility. Given that this training program includes training of attentional flexibility, we also expected a positive impact on switching. Indeed, being able to switch between two tasks implies being able to efficiently manage attentional resources. Being able to perform two tasks in parallel is difficult for older adult persons and implies being able to correctly manage the allocation of attention between the two tasks. Indeed, recruiting enough attentional resources and managing their allocation while ensuring the effectiveness and efficiency for both tasks is a key capacity to display good performance in dual tasks. Since our training included a dual task, we expected an improvement of this skill in participants. Based on the literature in older adult persons, planning could be particularly related to working memory. Since our training included a working memory task, it was of interest to evaluate the potential transfer to planning.

\section{Methods}

\section{Participants and screening for eligibility}

All participants provided written, informed consent according to procedures approved by the local Ethics Committee, which followed the recommendations of the Declaration of Helsinki. The participants were elderly volunteers with good cognitive status who lived independently in the community. They were recruited through flyers distributed by general practitioners, physiotherapists, associations for senior citizens, and social clubs and by personal contacts.

In the screening for eligibility, 53 volunteers were interviewed at home by a neuropsychologist during a 120-min session. During this session, the study plan and the research procedures were clearly explained. All the included subjects expressed their willingness to participate in the study and answered a questionnaire assessing their health status, medications, and educational level. Cognitive screening was performed to characterize the participants and exclude those with depression, mild cognitive impairment (MCI), or dementia. Volunteers were evaluated with the Mini-Mental State Examination (MMSE) [21], Clinical Dementia Rating (CDR) scale [22], Instrumental Activities of Daily Living (IADL) scale [23], and 30-item version of the Geriatric Depression Scale (GDS) [24].

Exclusion criteria for the study were (1) an MMSE score<27; (2) a CDR scale $\geq .5$; (3) abnormal activities of daily living, as assessed by the 4 items of the IADL scale; (4) a current depressive disorder, as indicated by a depression rating greater than 11 on the 30-item GDS; (5) a history of major systemic, psychiatric, or neurological disorder; (6) MCI, assessed by published criteria [25]; (7) any medications known to affect cognition; (8) severe auditory, visual, verbal, or motor deficits; and (9) recent participation in a cognitive training or neuropsychological research program.

Eight volunteers were excluded because of health problems or because they were unavailable during the study period. The 45 volunteers meeting the criteria were randomly assigned to one of the three experimental groups (15 subjects per group): (i) one group participating at home in the memory and attention training (Training group), (ii) one group participating at home in a paper-and-pencil game training (Leisure group), and (iii) one group that did not undertake any training (Control group). Table 1 shows that the mean demographic and clinical characteristics of participants did not differ between groups.

\section{Pretraining, posttraining and follow-up tasks}

Performances of the participants were evaluated using the trailmaking task (TMT) [26] (we considered the B-A score, which corresponds to the time needed to perform Part B of the task minus the time needed to perform Part A of the task); the dual task of Baddeley [27] (we considered the percentage of correct digit sequences and the number of boxes crossed in single and dual conditions); a 3-disk version of the Tower of Hanoi [28] (we considered the time (s) to solve the task and the number of moves); semantic and first letter fluency tasks of Cardebat [29] (we considered the number of words produced in $2 \mathrm{~min}$ ); and the Stroop interference test [30] (we considered the number of correctly named colors, name of colors and color of the ink used to write the words, each in $45 \mathrm{~s}$; each raw score was corrected for age following Golden norms [31]); the interference score was computed with the following formula [31]): Colored words raw - ((corrected Colors ${ }^{*}$ corrected Words)/(corrected Colors+ corrected Words)). When available, parallel versions of the tasks were used for the post-training and follow-up evaluations.

\section{Memory and attention training}

The training program used in the present study is the same as in Chambon et al. [7]. Succinctly, each training session was performed with the neuropsychologist and included a memory task and an attention task because of the close interaction between memory and attention processes.

To train memory, we used a visual recognition short-term memory (STM) task, a visuospatial recognition STM task, a visual working memory task, and a narrative memory task. During memory training, we improved encoding by working on mnemonic strategies. To improve information maintenance, we used the spaced retrieval method [32]. To improve resistance to interference, we introduced distractors between

Table 1. Mean demographics and clinical characteristics of the three participant groups. (Note: Training group=memory and attention training group; Leisure=paper-and-penci game training group. Control=nontraining group. ${ }^{\mathrm{a}} 1=$ primary school $/ 2=$ secondary school $/ 3=$ more than secondary school. MMSE=Mini-Mental State Examination. $\mathrm{CDR}=$ Clinical Dementia Rating. IADL=Instrumental Activities of Daily Living GDS $=$ Geriatric Depression Scale. Data are expressed as the mean \pm SEM; $n=15$ per group)

\begin{tabular}{|c|c|c|c|}
\hline Characteristics & Training group & Leisure group & Control group \\
\hline Age (year) & $74.5 \pm 2.1$ & $75.3 \pm 2.3$ & $74.4 \pm 3.4$ \\
Women/men & $9 / 6$ & $10 / 5$ & $9 / 6$ \\
Education (n) 1/2/3 & $6 / 5 / 4$ & $6 / 6 / 3$ & $7 / 5 / 3$ \\
MMSE (/30) & $28.9 \pm 0.3$ & $28.8 \pm 0.3$ & $29.0 \pm 0.2$ \\
CDR (/5) & $0 \pm 0$ & $0 \pm 0$ & $0 \pm 0$ \\
IADL (/4) & $0 \pm 0$ & $0 \pm 0$ & $0 \pm 0$ \\
30-item GDS (/30) & $3.1 \pm 0.3$ & $3.5 \pm 0.4$ & $3.2 \pm 0.3$ \\
\hline
\end{tabular}


the presentation of the material to memorize and the recognition test. Recognition was chosen to minimize processing at retrieval. Moreover, we use familiarity instead of recollection because it leads to less retrieval failures.

To train attention, we used a visual focused attention task, a visuospatial focused attention task and a dual task. During the attention training, we used focused and divided attention tasks based on the model of van Zomeren and Brouwer [33]. We also increased processing speed throughout training. Once the subjects had acquired the automatisms to properly perform the task, they were asked to increase their execution speed.

For a detailed description of the training tasks, see Supplementary Material.

\section{Paper-and-pencil game training}

This training consisted of games from magazines and newspapers. Each session was performed in presence of a neuropsychologist, using crosswords, arrow words, code words, Sudoku, games of visual discrimination consisting of finding resemblances and differences, hangman, games of logic, enigmas, labyrinths, tic-tac-toe, rebus, and puzzles. Tactics for each game were provided by the neuropsychologist to work on the subject's reasoning abilities.

Procedure: All participants were evaluated individually at home within a maximum of two weeks before the beginning of training (pretraining phase, T1), after the completion of training (posttraining phase, T2), and 6 months after the completion of training (follow-up phase, T3).

Training group: Before training, each participant was familiarized with the computer material, and completed a session to establish the baselines to be used at the beginning of the training.

Each participant took part in 24 individual, 1-h training sessions at home, 2 sessions per week for a period of 12 weeks, under the supervision of the neuropsychologist.

A typical training session consisted of three computer tasks: (i) a memory task; (ii) an attention task; and (iii) a systematic task of narrative memory. The organization of the training is described in the Supplementary Material. For the memory and attention tasks, the computer went through 4 mandatory steps assisted by the neuropsychologist: (1) Read general information regarding the trained memory or attention process; (2) Read the instructions for the task to be performed; (3) Perform some trials of the task. When the subject made an error, the computer provided the correct answer. The subject then had to provide the correct answer to continue the exercise; and (4) Perform the task and receive feedback by the neuropsychologist on their performance at the end of the exercise. Concerning the training of narrative memory, only steps (1), (2), and (4) were provided by the computer.

At the beginning of the memory-training tasks, the neuropsychologist proposed one encoding strategy to apply during the task, i.e., verbalization, mental imagery, association of ideas, and suggestions to imagine a story from the pictures shown or the words heard. At the beginning of the training of the dual task, the neuropsychologist made the subject aware of the higher attentional resources needed to perform the two tasks in parallel and of the need to properly allocate the necessary resources for each task. To do this, the subject listed the applicable strategies to best manage their attentional resources, and the neuropsychologist asked the subject to choose the one that best suits him. The procedure for setting up strategies to perform the dual task was as follows: 1) the subject became aware that his or her attentional resources was limited and that in a dual-task situation, he or she must share them; 2) this sharing must be done according to the subject's feeling on the 2 tasks, i.e., the subject must put more resources on the task that seems to him or her the most energy demanding; 3) the subject must choose a strategic by favoring time or accuracy, the right strategy being most often a compromise between the two; 4) as the subject progressed, he or she seeked to release attentional resources by setting up automatic procedures.

Whether it is for memory or attention exercises, the subject tested the chosen strategy by doing the exercise. At the end, each participant received individualized adaptive feedback about his performance. When performances revealed the use of an irrelevant strategy, a new one was proposed to the subject, and its efficacy was assessed by performing the task again.

The neuropsychologist individualized the training by modulating the difficulty of each task (increasing it when the performance level reached $90 \%$, or decreasing it, when the performance level was below $70 \%)$ so that the tasks would continue to be challenging. Task difficulty was modulated by the following parameters: picture display duration, number of pictures or words to remember, addition of a blank interval or a distractor between encoding and recognition, time interval between words, and choice of the target picture or target category.

Leisure group: All participants in the Leisure group performed the same sets of activities. The Leisure group was assigned the same number, frequency, and duration of training sessions as the Training group. The complexity of the games was also adapted by the neuropsychologist to the progress of the subject across sessions. A higher level was proposed when the neuropsychologist observed that the subject was comfortable with the game, e.g., correctly fill the grids up to $70 \%$ minimum and provided positive feedback.

Nontrained group (Control): Control participants did not take part in any training. They were tested according to the same neuropsychological schedule as the two other groups to check for possible retest effects.

Three neuropsychologists were involved in the study: one performed the screening for eligibility, one administered and scored assessments during the pre- and posttesting phases (this person was kept blind to the group membership of participants), and one supervised the training sessions.

\section{Statistical analysis}

Data are expressed as the mean \pm S.E.M. Statistical analyses were carried out using Statistica (Version 7.1, 2005) software. Analyses of variance (ANOVA) were used to compare baselines of subject characteristics, including age and screening inclusion measures. The results from each evaluation task and questionnaire were analyzed with repeated measures ANOVA. Subsequent post hoc Bonferroni-corrected for multiple comparisons t-tests were used. Effect sizes were calculated using $\eta_{\mathrm{p}}^{2}$; an $\eta_{\mathrm{p}}^{2}$ above 0.01 reflects a small effect, above 0.06 a medium effect, and above 0.14 a large effect) [34].

\section{Results}

\section{Tower of Hanoi test}

Repeated measures ANOVA on the time spent performing the Tower of Hanoï test showed a Testing time effect, $F(2,84)=22.89$, 
$\mathrm{p}<.001 ; \eta_{\mathrm{p}}^{2}=0.35$ (Table 2). This result indicate that all groups improved between $\mathrm{T} 1$ and $\mathrm{T} 2$.

Repeated measures ANOVA on the number of movements to perform the Tower of the Hanoï test showed no effect (Table 2).

\section{Fluency test}

Repeated measures ANOVA on the number of correct words for initial letter fluency showed a Testing time effect, $F(2,84)=3.40$, $\mathrm{p}=.038$; $\eta_{\mathrm{p}}^{2}=0.07$ (Table 2).

Repeated measures ANOVA on the number of correct words for semantic fluency showed a Group effect, $\mathrm{F}(2,42)=9.38, \mathrm{p}<.001 ; \mathrm{\eta}^{2} \mathrm{p}=0.31$, Testing time effect, $F(2,84)=16.42, \mathrm{p}<.001 ; \eta_{\mathrm{p}}^{2}=0.28$, and Group $\mathrm{x}$ Testing time interaction, $F(4,84)=5.35, \mathrm{p}<.001 ; \eta_{\mathrm{p}}^{2}=0.20$. Post hoc $\mathrm{t}$-tests showed that the Training group at T2 improved in performance compared to $\mathrm{T} 1$ and that this effect was maintained at T3. The Training group also improved at T2 compared to the Control and the Leisure groups (Figure 1).

\section{Stroop test}

Repeated measures ANOVA on the number of correctly read words showed no effect (Table 2). Repeated measures ANOVA on the number of correctly named colors showed no Group effect, a Testing time effect, $\mathrm{F}(2,84)=18.01, \mathrm{p}<.001 ; \eta_{\mathrm{p}}^{2}=0.30$, and a Group $\mathrm{x}$ Testing time interaction, $\mathrm{F}(4,84)=6.72, \mathrm{p}<.001 ; \mathrm{\eta}_{\mathrm{p}}^{2}=0.24$. Post hoc $\mathrm{t}$-tests showed that the Training group at $\mathrm{T} 2$ improved in performance compared to $\mathrm{T} 1$ and that this effect was maintained at T3 (all $p<.05$ ). A post hoc t-test also showed that the Leisure group improved at T2 compared to T1 (Figure 2A) $(p<.05)$.

Repeated measures ANOVA on the number of correctly identified colored words showed a Group effect, $\mathrm{F}(2,42)=15.38, \mathrm{p}<.001 ; \mathrm{\eta}_{\mathrm{p}}^{2}=0.42$,
Testing time effect, $F(2,84)=41.68, \mathrm{p}<.001 ; \mathrm{\eta}_{\mathrm{p}}^{2}=0.50$, and Group $\mathrm{x}$ Testing time interaction, $F(4,84)=16.31, \mathrm{p}<.001 ; \eta_{\mathrm{p}}^{2}=0.44$. Post hoc $\mathrm{t}$-tests showed that the Training group at $\mathrm{T} 2$ improved in performance compared to T1 and that this effect was maintained at T3. Moreover, the Training group performed better than both the Control and Leisure groups at $\mathrm{T} 2$ and $\mathrm{T} 3$, and the Leisure group improved at T2 compared to T1 (Figure 2B).

Repeated measures ANOVA on the interference score showed a Group effect, $\mathrm{F}(2,42)=7.85, \mathrm{p}=.0013 ; \eta_{\mathrm{p}}^{2}=0.27$, Testing time effect, $F(2,84)=16.41, \mathrm{p}<.001 ; \eta_{\mathrm{p}}^{2}=0.28$, and Group $\mathrm{x}$ Testing time interaction,

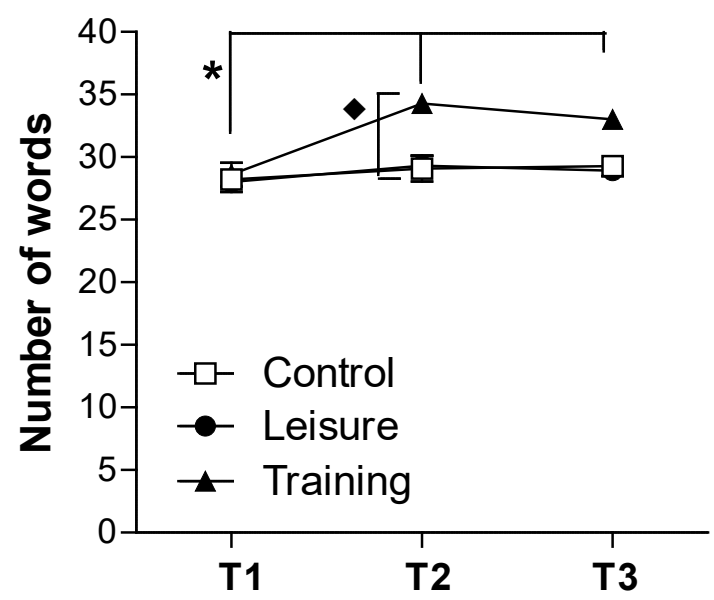

Figure 1. Effects of training on the semantic fluency test. The graph shows the number of correctly categorized words before training (T1), immediately post training (T2), and 6 months post training (T3). Data are expressed as the mean \pm SEM; $\mathrm{n}=15$ per group; ${ }^{*} p<.05$ between testing times for the Training group. ${ }^{*} p<.05$ between the Training group and other groups at a particular testing time

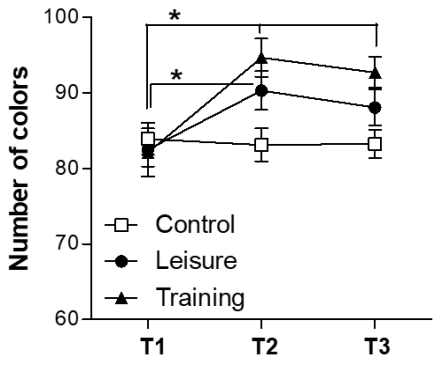

A.

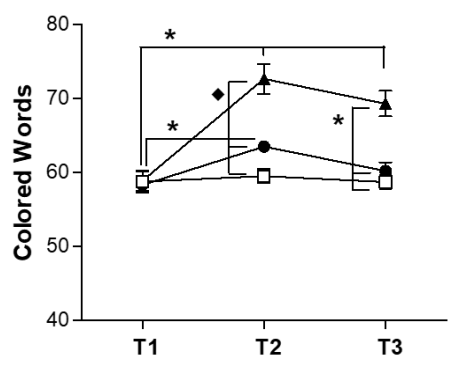

B.

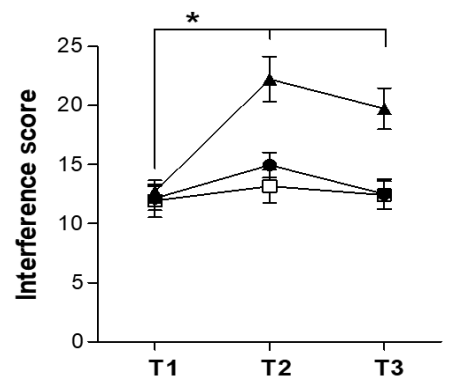

C.

Figure 2. Effects of training on the Stroop test. (A) The number of correctly named colors; (B) number of correctly named colored words; and (C) interference score. Data are expressed as the mean \pm SEM; $\mathrm{n}=15$ per group; ${ }^{*} p<.05$ between testing times for the Training group. ${ }^{*} p<.05$ between the Training group and other groups at a particular testing time

Table 2. Summary table of executive performances showing no benefit of training immediately after and at a 6-month follow-up

\begin{tabular}{|c|c|c|c|c|c|c|c|c|c|}
\hline \multirow[t]{2}{*}{ Task } & \multicolumn{3}{|c|}{ Control } & \multicolumn{3}{|c|}{ Leisure } & \multicolumn{3}{|c|}{ Training } \\
\hline & $\mathrm{T} 1$ & $\mathrm{~T} 2$ & $\mathrm{~T} 3$ & $\mathrm{~T} 1$ & $\mathrm{~T} 2$ & T3 & $\mathrm{T} 1$ & $\mathrm{~T} 2$ & T3 \\
\hline \multicolumn{10}{|l|}{ Tower of Hanö̈ } \\
\hline Time $(s)^{*}$ & $66.5 \pm 11.7$ & $40.9 \pm 3.7$ & - & $66.3 \pm 13.2$ & $40.1 \pm 3.9$ & - & $68.4 \pm 14.3$ & $19.7 \pm 4.7$ & - \\
\hline Movements & $9.0 \pm 0.5$ & $9.1 \pm 0.6$ & - & $9.1 \pm 0.6$ & $9.1 \pm 0.7$ & - & $9.3 \pm 0.8$ & $7.5 \pm 0.4$ & - \\
\hline \multicolumn{10}{|l|}{ Initial letter fluency } \\
\hline Words* & $21.7 \pm 1.1$ & $21.3 \pm 0.7$ & $21.1 \pm 0.6$ & $21.1 \pm 0.6$ & $22.2 \pm 1.4$ & $21.9 \pm 1.0$ & $22.1 \pm 1.2$ & $24.7 \pm 1.0$ & $23.7 \pm 1.0$ \\
\hline \multicolumn{10}{|l|}{ Stroop test } \\
\hline Read words & $106.5 \pm 2.0$ & $105.3 \pm 1.7$ & $105.1 \pm 1.6$ & $105.7 \pm 1.8$ & $105.6 \pm 1.7$ & $105.0 \pm 2.2$ & $106.2 \pm 1.5$ & $108.1 \pm 2.0$ & $107.0 \pm 1.8$ \\
\hline \multicolumn{10}{|l|}{ Dual task of Baddeley } \\
\hline Digit span & $5.2 \pm 0.2$ & $5.20 \pm 0.2$ & $5.4 \pm 0.1$ & $5.2 \pm 0.2$ & $5.2 \pm 0.1$ & $5.3 \pm 0.1$ & $5.2 \pm 0.2$ & $5.7 \pm 0.1$ & $5.4 \pm 0.1$ \\
\hline Box crossing single ${ }^{*}$ & $114.0 \pm 6.8$ & $111.60 \pm 4.7$ & $114.2 \pm 8.8$ & $112.6 \pm 7.3$ & $137.5 \pm 6.1$ & $120.1 \pm 4.8$ & $113.3 \pm 12.2$ & $127.5 \pm 6.6$ & $125.3 \pm 6.3$ \\
\hline
\end{tabular}

Note: The *indicates a Testing time effect $\mathrm{p}<0.05$. 
$F(4,84)=6.17, \mathrm{p}<.001 ; \eta_{\mathrm{p}}^{2}=0.23$. Post hoc $\mathrm{t}$-tests showed that the Training group at T2 improved in performance compared to T1 and that this effect was maintained at $\mathrm{T} 3$ (Figure 2C).

\section{Dual task of Baddeley}

Repeated measures ANOVA on the digit span showed no effect (Table 2). Repeated measures ANOVA on the correctly repeated digit sequences in the single-task condition showed a Group effect, $\mathrm{F}(2,42)=10.47, \mathrm{p}<.001 ; \eta_{\mathrm{p}}^{2}=0.33$, Testing time effect, $F(2,84)=5.99$, $p=.003 ; \eta_{\mathrm{p}}^{2}=0.12$, and Group $\mathrm{x}$ Testing time interaction, $F(4,84)=4.77$, $p=.002 ; \eta_{\mathrm{p}}{ }_{\mathrm{p}}=0.19$. Post hoc $\mathrm{t}$-tests showed that the Training group at $\mathrm{T} 2$ improved in performance, compared to $\mathrm{T} 1$ and to the Control group at $\mathrm{T} 2$ but also that the Training group at T3 showed improvements, compared to T1 (Figure 3A).

Repeated measures ANOVA on the correctly repeated digit sequences in the dual-task condition showed a Group effect, $\mathrm{F}(2,42)=8.99, \mathrm{p}<.001 ; \eta_{\mathrm{p}}^{2}=0.30$, Testing time effect, $F(2,84)=17.8$, $\mathrm{p}<.001 ; \mathrm{\eta}_{\mathrm{p}}^{2}=0.30$, and Group $\mathrm{x}$ Testing time interaction, $F(4,84)=14.37$, $\mathrm{p}<.001 ; \eta_{\mathrm{p}}^{2}=0.41$. Post hoc t-tests showed that performance of the Training group at $\mathrm{T} 2$ was improved compared to $\mathrm{T} 1$ and was better than both the Control and the Leisure groups at T2 (all $p<.05$ ). Furthermore, where an individual's performance in the Training group at T3 decreased significantly, compared to T2, they were nevertheless better than during their $\mathrm{T} 1$ performance (Figure 3B).

Repeated measures ANOVA on box crossing in the single-task condition showed a Testing time effect, $F(2,84)=6.65, \mathrm{p}=.002 ; \eta_{\mathrm{p}}^{2}=0.06$ (Table 3).

Repeated measures ANOVA on box crossing in the dual-task condition showed a Group effect, $\mathrm{F}(2,42)=4.12, \mathrm{p}=.023 ; \eta_{\mathrm{p}}^{2}=0.16$, Testing time effect, $F(2,84)=5.00, \mathrm{p}=.009 ; \mathrm{\eta}_{\mathrm{p}}^{2}=0.11$, and Group $\mathrm{x}$ Testing time interaction, $F(4,84)=3.89, \mathrm{p}=.006 ; \mathrm{\eta}_{\mathrm{p}}^{2}=0.16$. Post hoc $\mathrm{t}$-tests showed that the Training group at $\mathrm{T} 2$ improved in performance compared to $\mathrm{T} 1$ and that this effect was maintained at T3 (Figure 3C).

Repeated measures ANOVA on the TMT (B-A score) showed a Group effect $F(1,2)=4.87, \mathrm{p}=.012 ; \eta_{\mathrm{p}}^{2}=0.13$ (Figure 4).

\section{Discussion}

The data after training showed benefits in the Stroop test, semantic fluency test, dual task of Baddeley, and TMT. At the 6-month follow-up, all benefits were still present. No benefits were found in the initial letter fluency test or in the Tower of Hanoi test.

\section{Absence of benefits}

Our training did not improve performance in the initial letter fluency task. This can be explained by the fact that healthy elderly do not display any clear deficit in this task, even later in age, e.g., between 72 and 95 years [35]. Thus, our training cannot improve a performance that is high, if not already optimal.

We also observed no significant effect of our training on planning capacities, as assessed with the Tower of Hanoï task, even though there were clear tendencies for improvement in both measures (the solving time decreased from $68.4 \pm 14.29 \mathrm{~s}$ to $19.73 \pm 4.68 \mathrm{~s}$ and the number of movements decreased from $9.27 \pm 0.89$ to $7.53 \pm 0.41$; note that 7 is the minimal number of movements that can be performed in this version of the task). Further experiment needs to be performed in order to check whether this tendency reflects a potential effect of not.

\section{Benefits on the resistance to interference}

In the Stroop test, which requires effortful attention or controlled processing to perform correctly, executive control mechanisms are engaged (for review see [36]) and the inhibition required to deliberately stop a response that is relatively automatic is needed. In the present study, Training subjects were more resistant to interference than the other groups (Figure 2) and they were more able to inhibit irrelevant stimuli. Note that in the Leisure group, the interference score was not improved after the training period. Taken together, these results indicated that practicing leisure-type cognitive activities was not sufficient to improve the highly controlled process that is resistant to interference.

Since we trained resistance to interference via use of distractors and updating, we propose that this better performance of the Training group reflected a near transfer of benefit in a trained cognitive ability but into a new cognitive context. Our results agreed with those reported by Sandberg et al. [37] following executive training focusing on updating, shifting, and inhibition in older adults. Additional support can be found in a study from Borella et al. [38] investigating the benefits of working memory training in adults aged 65-75 years. Indeed, the authors reported transfer effects for tasks that represented the same narrow ability, different narrow but same broad ability, and different abilities. Borella et al. [38] highlighted that their training tasks engaged multiple processes, including the encoding and maintaining information, inhibiting no-longer-relevant information, simultaneous managing two tasks, shifting attention, and controlling attention. This was, for them, the reason their training could induce transfer effects.

\section{Digit sequence Single task condition}

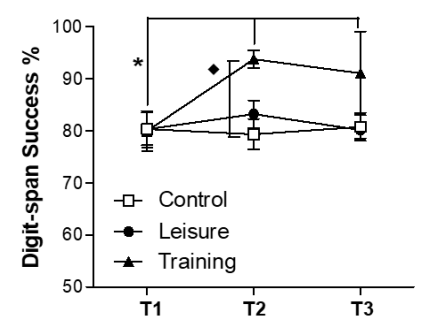

A.

\section{Digit sequence Dual task condition}

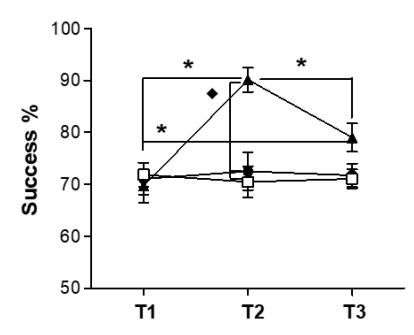

B.

\section{Box crossing Dual task condition}

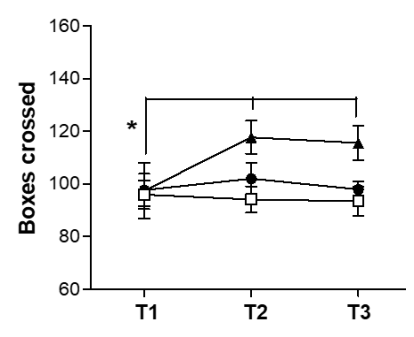

C.

Figure 3. Effects of training on the dual task of Baddeley. (A) Percentage of correct digit sequences in the single-task condition; (B) percentage of correct digit sequences in the dual-task condition; and $(\mathrm{C})$ number of boxes crossed in the dual-task condition. Data are expressed as the mean $\pm \mathrm{SEM} ; \mathrm{n}=15$ per group; * $p<.05$ between testing times for the Training group. ${ }^{*} p<.05$ between the Training group and other groups at a particular testing time 


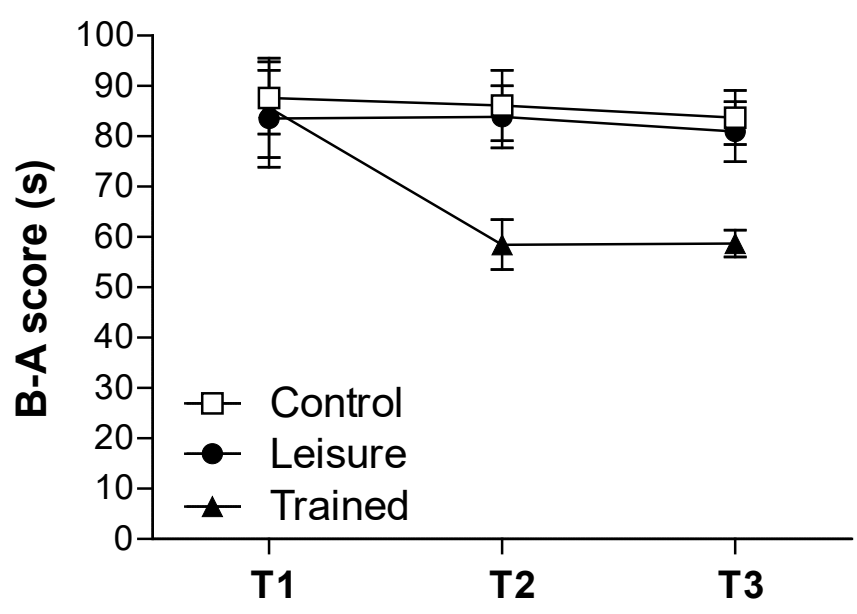

Figure 4. Effects of training on the trail-making test. The B-A score is given in seconds, before training (T1), immediately post training (T2), and 6 months post training (T3). Data are expressed as the mean \pm SEM; $n=15$ per group. Statistical analysis indicated a group effect

Thus, despite the differences between the two training programs, it is feasible that both Borella et al. [38] and our study showed improvements in the same cognitive ability of resistance to interference because both studies stimulated similar cognitive abilities.

\section{Benefits on attentional sharing}

In the dual-task condition of the dual task of Baddeley (Figure 3 ), we reported that the Training group managed the two tasks at the same time as easily as each single task, whereas the Control and Leisure groups had difficulties. These results showed that our training improved the attentional sharing capacity of the elderly participants, as a direct benefit from our training program, which included a dual task. Moreover, since the training dual task was a "cognitive-cognitive" task, whereas the evaluation dual task was a "cognitive-sensorimotor" task, such improvement showed that the Training group could transfer the knowledge acquired during training to a new dual-task situation. This can be explained by the fact that the strategies implemented by the subjects during the training dual task can be applied to any cognitive task requiring a high mental load. These strategies allowed the subject to have a metacognitive approach and optimal control of the distribution of his or her attentional resources between the two tasks.

Our results confirm data from the literature showing that after a dual-task training, participants transferred the time-sharing skills acquired and applied them to a new set of tasks [39]. In older adults, the efficiency of dual-task training has been reported in many studies. Bherer et al. [40] reported that a dual-task training (five training sessions that included a visual discrimination task and an auditory task) improved performance and induced a transfer to other dual tasks (i.e., a new visual-auditory task with new material and two visual-visual tasks). Taken together, these results and our present results indicated that when participants were trained in a dual task, elderly subjects can not only learn to perform better in the trained task but also acquire specific skills that can be used in other dual-task situations.

\section{Benefits on switching}

We observed a decreased B-A score in the Training group (Figure 4) that indicated a benefit of our training on the ability to switch efficiently. In a study from Sánchez-Cubillo et al. [41], this score was identified as the better indicator of executive control abilities, minimizing visuoperceptual and working memory demands. The main contributory variable that explained these findings was identified as the cost of switching, measured as the difference between switch and nonswitch trials $[42,43]$. We also observed better performances in the Training group on the semantic fluency test (Figure 1). Equivalent results were reported by Youn et al. [44] after a multistrategic memory training, including a metamemory approach, used in elderly participants to improve knowing, monitoring and judging their memory. Semantic fluency appears to be age sensitive, whereas first letter fluency is not $[45,46]$. An explanation proposed to this difference is that since letter fluency only relies on clustering when semantic fluency relies on clustering and switching, elderly adults tend to switch less frequently than younger adults to find a new category, which reflects a decline in strategic search processes [47].

The better performance on the TMT and semantic fluency test suggested that our training could improve switching capacity, thus showing a transfer of knowledge to a nontrained task. We think that 1) training working memory has greatly helped to improve switching by training inhibition and updating, and 2) training attentional flexibility, via our visuospatial focused attention task, had a positive impact on switching. With regard more specifically to semantic fluency, training encoding through memorization strategies may have had positive effects on switching and processing speed when the task was to actively search in memory for words belonging to a given category.

In addition, the results from the Training and Leisure groups indicated that to improve semantic fluency, cognitive training needs to provide "cognitive tools" that can be used by the elderly subjects outside of the training context and that manipulating words in various games is not sufficient for the transfer of learning to semantic fluency capacity.

Since the switching capacity was indirectly trained by providing memorization strategies to the elderly participants during training, our results can be interpreted as a near transfer of the benefits of a trained ability, i.e., switching, into new task contexts.

\section{Maintenance of benefits}

At the 6-month follow-up, all executive benefits were still present. The maintenance of cognitive benefits, where no booster session was conducted between the end of training and the follow-up evaluation, may have suggested that the benefits were not simply the result of repetitive practice during training. A coherent explanation could be that the strategies learned to resist interference, to inhibit and to manage attentional resources in the dual-task condition, in addition to their manipulation during training, favored their use in everyday life. Thus, the Training subjects may have used these capacities regularly, which must have allowed them to maintain a good level of performance 6 months later. Unfortunately, we did not quantify this transfer in everyday life and can thus not confirm this assumption. Another explanation could be that a multidomain training like our training favored relationships between mental processes and thus induced long-lasting modifications in brain functioning. Indeed, supporting this hypothesis, Cheng et al. [48] reported that multidomain cognitive training showed increased maintenance of the training effects at a 6-month follow-up compared to single-domain training in the elderly participants.

\section{Conclusion}

The present results taken with those from Chambon et al. [7] highlighted a large range of benefits, from memory to executive functioning and self-esteem, after the multifactorial training program 
we used with the older adult participants. Thanks to the Leisure group performances, these benefits can be attributed to the structure and personalization of the training while excluding the impact of a regular nonspecific interaction with a trainer. Moreover, the maintenance of benefits to 6 months indicated that trained subjects accommodated their new abilities acquired during training. In the future, we plan to modify this training for use in the early stage of Alzheimer's disease. Indeed, we aim to develop a cognitive program that can be adapted to the entire range of aging populations, i.e., from the least to the most cognitively affected older adult populations.

\section{Supplemental material}

Training was computer based, a format known to be beneficial to both young and older persons (Rasmusson et al., 1999). Training exercises were programmed in Java (Release 1.4) and conducted on a Microsoft Windows-based personal computer with a 12-in screen and a resolution of 1,024 x 768 pixels. Stimuli used were pictures of $256 \times 256$ pixels in categories such as animals, flowers, complex scenes, objects of everyday life, and common words pronounced by the computer. Using these stimuli is advantageous because they activate distinct regions. Verbal material encoding activates left frontal areas and picture encoding paradigms often activate both hemispheres in frontal areas (Golby et al., 2001; Kelley et al., 1998). Those studies showed increased encoding requirements for pictures, or the dual coding of pictures in visual and verbal formats. In addition, pictures, particularly complex scenes, strongly activate hippocampal and parahippocampal structures (Henke, Buck, Weber, \& Wieser, 1997).

\section{The memory training tasks}

Responses to these training tasks were given using a computer mouse.

Visual recognition short-term memory (STM) training task: The task lasted $5 \mathrm{~min}$. Participants were trained with three variants of the task. In Variant 1, they were asked to memorize pictures and to recognize them among lures immediately after presentation. They had to click on the recognized pictures. Variant 2 was identical to Variant 1 , but memorization and recognition were separated by a blank interval of 10,30 , or $60 \mathrm{~s}$. Variant 3 was identical to Variant 2 but a distractor, that is, a multiple-choice questionnaire, was introduced during the interval. We determined the percentage of correct responses.

Visuospatial recognition STM training task: The task lasted 5 min. Participants were trained with three variants of the task. In Variant 1 , they had to memorize the position of three to six identical images on the screen and then to recognize this layout among two propositions: one was the correct layout and the other one was different. Participants had to click on the correct one. Variant 2 was identical to Variant 1 , but memorization and recognition were separated by a blank interval of 10, 30, or $60 \mathrm{~s}$. Variant 3 was identical to Variant 2, but a multiple choice questionnaire distractor was introduced during the interval. We determined the percentage of correct responses.

Visual working memory training task: The task lasted $5 \mathrm{~min}$. At the beginning of the task, participants had to memorize pictures; in the next trials, they were asked to recognize the previously presented pictures (by clicking on them) and to memorize novel ones. Thus,

Table 3. Training organization. Note: Training consisted of 24 sessions. Memory and attention processes were concurrently stimulated. Each session included an attention task, a short-term memory task, and a narrative task. Training of memory promoted stimulation of visual (V) and visuospatial (VS) modalities alternatively by using an adaptation of the spaced retrieval method. Training of attention was based on a specific approach that considered the sub-components of attention as being distinct, hierarchical, and independent. Concerning the selectivity, we articulated our training on selective visual and visuospatial attention, divided attention (dual task), and working memory (WM). This intensity aspect of attention was managed by manipulating attentional load. For each hierarchical step of the selectivity, hierarchical steps of the intensity were embedded.Variant 1 (V1), Variant 2 (V2), Variant 3 (V3)

\begin{tabular}{|c|c|c|c|c|c|c|c|c|}
\hline \multicolumn{9}{|c|}{ Training organization } \\
\hline \multirow[b]{2}{*}{ Session } & \multicolumn{4}{|c|}{ Short-term memory } & \multirow[t]{2}{*}{ WM } & \multirow[t]{2}{*}{ NM } & \multicolumn{2}{|c|}{ Attention Dual task } \\
\hline & Version & Target & Delay & Distractor & & & Version & \\
\hline 1 & $\mathrm{v}-\mathrm{V} 1$ & Pictures & & & & 1 & $\mathrm{v}-\mathrm{V} 1$ & \\
\hline 2 & vs-V1 & Layout & & & & 2 & $\mathrm{v}-\mathrm{V} 1$ & \\
\hline 3 & & & & & Yes & 3 & vs-V1 & \\
\hline 4 & $\mathrm{v}-\mathrm{V} 2$ & Pictures & $10 \mathrm{~s}$ & & & 4 & vs-V1 & \\
\hline 5 & vs-V2 & Layout & $10 \mathrm{~s}$ & & & 5 & & Yes \\
\hline 6 & & & & & Yes & 6 & & Yes \\
\hline 7 & $\mathrm{v}-\mathrm{V} 2$ & Pictures & $30 \mathrm{~s}$ & & & 7 & vs-V1 & \\
\hline 8 & vs-V2 & Layout & $30 \mathrm{~s}$ & & & 8 & vs-V2 & \\
\hline 9 & & & & & Yes & 9 & & Yes \\
\hline 10 & $\mathrm{v}-\mathrm{V} 3$ & Pictures & $10 \mathrm{~s}$ & MCQ & & 10 & & Yes \\
\hline 11 & $\mathrm{v}-\mathrm{V} 3$ & Pictures & $10 \mathrm{~s}$ & MCQ & & 11 & $\mathrm{v}-\mathrm{V} 2$ & \\
\hline 12 & $\mathrm{v}-\mathrm{V} 2$ & Pictures & $30 \mathrm{~s}$ & & & 12 & vs-V2 & \\
\hline 13 & & & & & Yes & 13 & & Yes \\
\hline 14 & vs-V2 & Layout & $30 \mathrm{~s}$ & & & 14 & $\mathrm{v}-\mathrm{V} 2$ & \\
\hline 15 & $\mathrm{v}-\mathrm{V} 2$ & Pictures & $30 \mathrm{~s}$ & & & 15 & & Yes \\
\hline 16 & & & & & Yes & 16 & vs-V2 & \\
\hline 17 & vs-V3 & Layout & $30 \mathrm{~s}$ & MCQ & & 17 & $\mathrm{v}-\mathrm{V} 2$ & \\
\hline 18 & & & & & & 18 & vs-V3 & \\
\hline 19 & & & & & Yes & 19 & vs-V3 & \\
\hline 20 & $\mathrm{v}-\mathrm{V} 2$ & & $60 \mathrm{~s}$ & & & 20 & & Yes \\
\hline 21 & vs-V2 & Layout & $60 \mathrm{~s}$ & & & 21 & vs-V3 & \\
\hline 22 & & & & & Yes & 22 & $\mathrm{v}-\mathrm{V} 2$ & \\
\hline 23 & $\mathrm{v}-\mathrm{V} 3$ & Pictures & $60 \mathrm{~s}$ & MCQ & & 23 & & Yes \\
\hline 24 & vs-V3 & Layout & $60 \mathrm{~s}$ & MCQ & & 24 & vs-V3 & \\
\hline
\end{tabular}


participants had to memorize (new) and recognize (old) pictures in the same trial. This procedure was repeated throughout the task, so that in the next trial, the new pictures became the old pictures and so on. Such task solicits up-dating processes at each trial in order to make a new picture an old one while making space for the next new picture. In this task we determined the percentage of correct responses.

Narrative memory training task: A story with 24 episodes was told at the rate of one episode per training session. After the first session, a question was asked about the episode. The subject chose the answer among three choices. Only one was the correct response. After the second session, two questions were asked: one evaluating memory of the previous episode, and one assessing memory of the new episode. From the third training session, participants had to answer a question about one of the learned episodes (remote memory), a question about the previously learned episode (recent memory), and a question on the newly learned episode (immediate memory). If the participants made a mistake, they could read the corresponding episode. We determined the percentage of correct responses.

\section{The attention training tasks}

Responses to these training tasks were given using a standard keyboard (using only 2 keys).

Visual focused attention training task: The task lasted $3 \mathrm{~min}$. Participants were trained with three variants of the task. In Variant 1, they had to focus their attention on the middle of the screen to detect a target picture. Pictures (50\% target, 50\% lures) appeared one after the other in the middle of the screen. Participants had to press on the key "enter" to answer "yes, it is the target," and on the key "esc" to answer "no, it is not the target." Participants were instructed to respond as fast and as accurately as possible. In Variant 2, the target was a category. We determined the percentage of correct responses and mean response time (seconds).

Visuospatial focused attention training task: The task lasted 3 min. Participants were trained with three variants of the task. In Variant 1 , pictures (50\% target, $50 \%$ lures) appeared one after the other on the left or the right side of the screen. Participants had to press a key "enter" to answer "yes, it is the target," and on the key "esc" to answer "no, it is not the target." They were instructed to respond as fast and as accurately as possible. In Variant 2, two pictures appeared at the same time; one was pointed to by an arrow. The participants had to respond if the picture pointed to by the arrow was the target picture or not. They were instructed to ignore pictures that were not pointed to by the arrow even if they were the target picture. Variant 3 was identical to Variant 2, but the arrow appeared only to indicate a change of side. When no arrow was present, participants had to continue to answer on the same side of the screen, until a new arrow appeared. We determined the percentage of correct responses and mean response time (seconds).

Dual-task: The task was composed of six trials. Participants were asked to concurrently perform one auditory STM task and the visualfocused attention task described previously. The dual task consisted in memorizing words pronounced by the computer, while performing the visual-focused attention task, and at the end of the trial in recognizing, among lures, pictures matching the words heard. We determined the percentage of correct responses for the auditory short-term memory and the percentage of correct responses, as well as the mean response time (seconds) for the visual-focused attention. Modulated parameters and cognitive processes trained are detailed in our previous publication (Herrera et al., 2012).

\section{Acknowledgment}

This study was financially supported by the Centre National de la Recherche Scientifique (CNRS) but not associated to any specific grant.

\section{Conflicts of interest}

The authors disclaim no conflict of interest.

\section{References}

1. Belleville S, Gilbert B, Fontaine F, Gagnon L, Ménard E, et al. (2006) Improvement of episodic memory in persons with mild cognitive impairment and healthy older adults: evidence from a cognitive intervention program. Dement Geriatr Cogn Disord 22: 486499. [Crossref]

2. Craik FI, Winocur G, Palmer H, Binns MA, Edwards M, et al. (2007) Cognitive rehabilitation in the elderly: effects on memory. J Int Neuropsychol Soc 13: 132-142. [Crossref]

3. Neely AS and Backman L (1995) Effects of multifactorial memory training in old age: generalizability across tasks and individuals. J Gerontol B Psychol Sci Soc Sci 50: 134-140.

4. Stigsdotter Neely A (2000) Multifactorial memory training in normal aging: In search of memory improvement beyond the ordinary. In D.R. Hill, L. Bäckman, A. StigsdotterNeely (Eds.), Cognitive rehabilitation in old age (pp.63-80). New York, NY: Oxford University Press.

5. Levine B, Stuss DT, Winocur G, Binns MA, Fahy L, et al. (2007) Cognitive rehabilitation in the elderly: Effects on strategic behavior in relation to goal management. $J$ of the Intl Neuropsychol Society 13(01).

6. Schmiedek F, Lövdén M, Lindenberger U (2010) Hundred days of cognitive training enhance broad cognitive abilities in adulthood: Findings from the COGITO study. Front Aging Neurosci 2. [Crossref]

7. Chambon C, Herrera C, Romaiguere P, Paban V, Alescio-Lautier B, et al. (2014) Benefits of computer-based memory and attention training in healthy older adults. Psychology and Aging 29: 731-743.

8. Norman DA \& Shallice T (1986) Attention to action: Willed and automatic control of behavior. In Consciousness and self-regulation: Advances in research (In R. J. Davidson \& G. E. Schwartz \& D. Shapiro (Eds.), Vol. IV).

9. Stuss DT (1992) Biological and psychological development of executive functions. Brain Cogn 20: 8-23. [Crossref]

10. Sorel O, Pennequin V (2008) Aging of the planning process: the role of executive functioning. Brain Cogn 66: 196-201. [Crossref]

11. Kostering L, Leonhart R, Stahl C, Weiller C, Kaller CP, et al. (2016) Planning decrements in healthy aging: mediation effects of fluid reasoning and working memory capacity. The Journals of Gerontology Series B: Psychological Sciences and Social Sciences 71: 230-242.

12. Verhaeghen P, Steitz DW, Sliwinski MJ, Cerella J (2003) Aging and dual-task performance: A meta-analysis. Psychology and Aging 18: 443-460.

13. Verhaeghen P, Cerella J (2002) Aging, executive control, and attention: a review of meta-analyses. Neuroscience and Biobehavioral Reviews 26: 849-857.

14. Wasylyshyn C, Verhaeghen P, Sliwinski MJ (2011) Aging and task switching: a metaanalysis. Psychol Aging 26: 15-20. [Crossref]

15. Bugg JM, DeLosh EL, Davalos DB, Davis HP (2007) Age differences in Stroop interference: contributions of general slowing and task-specific deficits. Neuropsychol Dev Cogn B Aging Neuropsychol Cogn 14: 155-167. [Crossref]

16. Davidson DJ, Zacks RT, Williams CC (2003) Stroop interference, practice, and aging. Neuropsychol Dev Cogn B Aging Neuropsychol Cogn 10: 85-98. [Crossref]

17. Fjell AM, Sneve MH, Grydeland H, Storsve AB, Walhovd KB, et al. (2016) The disconnected brain and executive function decline in aging. Cerebral Cortex 27: 23032317.

18. Mayas J, Fuentes LJ, Ballesteros S (2012) Stroop interference and negative priming (NP) suppression in normal aging. Arch Gerontol Geriatr 54: 333-338. [Crossref]

19. Andrés P, Guerrini C, Phillips LH, Perfect TJ (2008) Differential effects of aging on executive and automatic inhibition. Developmental Neuropsychology 33: 101-123.

20. Belleville S, Rouleau N, Van der Linden M (2006) Use of the Hayling task to measure inhibition of prepotent responses in normal aging and Alzheimer's disease. Brain Cogn 62: 113-119. [Crossref] 
21. Folstein MF, Folstein SE, McHugh PR (1975) "Mini-mental state." Journal of Psychiatric Research 12: 189-198.

22. Hughes CP, Berg L, Danziger WL, Coben LA, Martin RL, et al. (1982) A new clinical scale for the staging of dementia. The British Journal of Psychiatry 140: 566-572.

23. Lawton MP, Brody EM (1969) Assessment of older people: Self-maintaining and instrumental activities of daily living. The Gerontologist 9 (3 Part 1): 179-86.

24. Yesavage JA, Brink TL, Rose TL, Lum O, Huang V, et al. (1982) Development and validation of a geriatric depression screening scale: a preliminary report. J Psychiatr Res 17: 37-49. [Crossref]

25. Petersen RC, Smith GE, Waring SC, Ivnik RJ, Tangalos EG, et al. (1999) Mild cognitive impairment: clinical characterization and outcome. Arch Neurol 56: 303-308. [Crossref]

26. Reitan RM (1958) Validity of the trail making test as an indication of organic brain damage. Perceptual and Motor Skills 8: 271-278.

27. Baddeley A, Della Sala S, Papagno C, Spinnler H (1997) Dual-task performance in dysexecutive and nondysexecutive patients with a frontal lesion. Neuropsychology 11: 187-194.

28. Simon H (1975) The functional equivalence of problem solving skills. Cognitive Psychology 3: 268-288.

29. Cardebat D, Doyon B, Puel M, Goulet P, Joannette Y, et al. (1990) The literal and categorical verbal fluency of Cardebat.

30. Stroop R (1935) Studies of interference in serial verbal reactions. Journal of Experimental Psychology 4: 643-663.

31. Golden C (1978) Stroop color and word test. Wood Dale, IL: StoeltingCo.

32. Landauer TK, Bjork RA (1978) Optimum rehearsal patterns and name learning. In M. M. Gruneberg, P. E. Morris, \& R.N. Sykes (Eds.), Practical aspects of memory (pp.625-632). New York, NY: Academic Press.

33. van Zomeren AH, Brouwer WH (1994) Clinical neuropsychology of attention. NewYork, NY: Oxford University Press.

34. Cohen J (1988) Statistical power analysis for the behavioral sciences (San Diego, CA Academic Press.).

35. Bryan J, Luszcz MA, Crawford JR (1997) Verbal knowledge and speed of information processing as mediators of age differences in verbal fluency performance among older adults. Psychology and Aging 12: 473-478.
36. MacLeod CM (1992) The Stroop task: The "gold standard" of attentional measures. Journal of Experimental Psychology 121: 12-14.

37. Sandberg P, Ronnlund M, Nyberg L, Stigsdotter Neely A (2014) Executive process training in young and old adults. Aging, Neuropsychology, and Cognition 21: 577-605.

38. Borella E, Carretti B, Riboldi F, De Beni R (2010) Working memory training in older adults: Evidence of transfer and maintenance effects. Psychology and Aging 25: 767778 .

39. Damos DL, Wickens CD (1980) The identification and transfer of timesharing skills. Acta Psychologica 46: 15-39.

40. Bherer L, Kramer AF, Peterson MS, Colcombe S, Erickson K, et al. (2005) Training effects on dual-task performance: are there age-related differences in plasticity of attentional control? Psychol Aging 20: 695-709. [Crossref]

41. Sánchez-Cubillo I, PerianEz JA, Adrover-Roig D, RodríGuez-SáNchez JM, RíOs-Lago M, et al. (2009) Construct validity of the trail making test: Role of task-switching, working memory, inhibition/interference control, and visuomotor abilities. Journal of the International Neuropsychological Society 15: 438.

42. Allport A, Styles EA, Hsieh S (1994) Shifting intentional set? Exploring the dynamic control of tasks. Attention and performance (En: M. Moscovitch (ed.), Vol. XV, pp. 421-452).

43. Rogers RD, Monsell S (1995) Costs of a predictible switch between simple cognitive tasks. Journal of Experimental Psychology 124: 207-231.

44. Youn JH, Lee JY, Kim S, Ryu SH (2011) Multistrategic memory training with the metamemory concept in healthy older adults. Psychiatry Investig 8: 354-361. [Crossref]

45. Mejia S, Pineda D, Alvarez LM, Ardila A (1998) Individual differences in memory and executive function abilities during normal aging. The International Journal of Neuroscience 95: 271-284.

46. Bryan J, Luszcz MA (2000) Measurement of executive function: Considerations for detecting adult age differences. Journal of Clinical and Experimental Neuropsychology 22: $40-55$.

47. Troyer AK, Moscovitch M, Winocur G (1997) Clustering and switching as two components of verbal fluency: evidence from younger and older healthy adults. Neuropsychology 11: 138-146.

48. Cheng Y, Wu W, Feng W, Wang J, Chen Y, et al. (2012) The effects of multi-domain versus single-domain cognitive training in non-demented older people: A randomized controlled trial. BMC Medicine 10.

Copyright: (C2019 Chambon C. This is an open-access article distributed under the terms of the Creative Commons Attribution License, which permits unrestricted use, distribution, and reproduction in any medium, provided the original author and source are credited. 